\title{
Plight of British postdocs
}

SIR-The recent report on the plight of older postdoctoral scientists in the United Kingdom (Nature 323, 8; 1986) will come as no surprise to most university researchers. The reasons for the discrimination against older (over 30 ) postdocs are quite straightforward. First, it can be more than 30 per cent more expensive to employ postdocs in their early $30 \mathrm{~s}$ compared to newly qualified $\mathrm{PhDs}$ in their mid-20s. This is a real problem for academics who may wish or even prefer to employ a more experienced and mature postdoctoral assistant. It is difficult to justify this extra salary to the research councils; it is now common to be told when a grant is reviewed that only a postdoc at the lower end of the salary scale should be employed.

Second, older postdocs are less likely to remain for the full three-year tenure of most research council grants. The older a postdoc, the more experience he (or she) will have and the more anxious he will be to find a more secure job, or even to emigrate. This is especially true because he realizes that the older he is, the less employable he will be as a postdoc. The pragmatic academic will therefore tend to be biased towards the selection of more youthful applicants for postdoctoral positions.

Like many scientists of my generation, I found myself obliged to take on a series of short-term postdoctoral contracts of between one and three years' duration. During this time, I spent three years in the United Kingdom, one year each in West Germany and Australia and three years in the United States. These moves were not made out of any particular wanderlust on my part but were made necessary as each short-term contract expired.

One obvious solution is to abolish agerelated salary scales for postdocs. In the United States there are no such scales. Salaries vary from university to university, and postdocs in their 40 s are often paid the same salary (and do the same job) as those in their 20s. In West Germany, the equivalent of postdocs (wissenschaftliche Assistenten) are not on an age-related scale. While such a measure would remove the postdoc age anomaly, it would probably not entirely halt the increasing drain of scientific talent away from these shores. However, the brain drain occurs at all levels, from graduate students to professors, and is mostly due to the greater scientific and pecuniary opportunities that exist abroad.

The recent reductions in the numbers and quality of new postdocs coming onto the market, coupled with a virtual cessation of recruitment for lectureships, has produced a large 'bulge' of postdocs in their 30s. This represents a formidable pool of talent for the nation that ought not to be allowed to go to waste. Many of us would be only too happy to employ older postdocs but find our hands tied by the present rigid and outmoded salary scale.

Denis J. Murphy

University of Durham,

Department of Botany,

Science Laboratories,

South Road, Durham DH1 3LE, UK

\section{Primitive myth}

SIR-It is a myth that preindustrial civilizations lived in harmony with nature, Jared Diamond writes in News and Views'. His argument is unassailable. But by dwelling exclusively on instances of bad natural resource management, he runs the risk of perpetrating a countermyth that sound environmental practices were absent in such civilizations.

Diamond draws a number of his examples of ecological mismanagement from the islands of Oceania. What he does not mention, however, is the fact that Pacific islanders were far ahead of Western industrial civilizations in some forms of environmental management. For example, they devised and practised a wide range of marine fisheries conservation measures long before they were first used in European civilizations.

Closed seasons, closed areas, size restrictions and limited entry have been widely employed in Polynesia and Micronesia for centuries as deliberate means of preventing overfishing; the need for conservation of marine fish stocks was first recognized in Great Britain little more than 80 years ago ${ }^{2}$.

To be sure, various instances of marine environmental misuse have also been documented in Pacific island cultures ${ }^{2}$. But the existence of the latter does not diminish the significance of the former. The coexistence of wise and unsound environmental practices is a characteristic of many if not all civilizations.

The danger of overemphasizing the weaknesses of environmental management in preindustrial cultures is that their strengths will be ignored. This issue is not one of mere historical accuracy. Unique environmental knowledge and timetested environmental management practices can still be found in some of these cultures, especially in the tropics where western scientific knowledge of natural resources is weakest.

Both UNESCO and IUCN have recognized the value of traditional knowledge and management of natural resources as a complement to existing scientific knowledge. Both organizations have recently set up working groups specifically to investigate its contemporary value. The re- cording and evaluation of such information is an urgent matter. It is disappearing rapidly as a result of the impact on native cultures of Western civilization.

R.E. JOHANnES

CSIRO Division of Fisheries Research,

Box 1538, Hobart,

Tasmania 7001, Australia

1. Diamond, J.M. Nature 324, 19 (1986).

2. Johannes, R.E. A. Rev. Ecol. Syst. 9,349 (1978).

\section{Art of citefaction}

SIR-In a recent leading article (Nature 324,$95 ; 1986$ ) you mention the possibility of citation counts being used to measure the research effectiveness of a university. Citations counts are important for other purposes and will appear to politicians to be a natural choice for this purpose, so they may well come to be used. But one consequence of this would be a considerable increase in citefaction - working to increase one's citesum - rather than research.

As in some other respects, UK academics are amateurs in citefaction. Many work in areas such as arts, basic science or engineering instead of in biochemistry or medicine where there are so many more active authors in the world that citesums are naturally much greater. Some even collaborate with industry in work that may not be publishable. Papers get published in journals with a low 'impact factor' (most UK ones). Too many try to keep references to a minimum by lazily using review articles 'where earlier references may be found' instead of listing them in full.

Citations are of two kinds. When an author references his own work, or that of a colleague, this is an 'incitation'. Incitation is largely within the control of the group and, if done systematically, can increase quadratically with the number of publications of the group whereas 'excitation' tends to increase linearly .

If the vice-chancellors are serious in using these counts they will need to solve some complicated issues. Citations of papers can behave quite differently. Some peak quickly but others rise slowly over a long period to great heights. One, last year, reached over 6,000 citations after a steady rise through 15 years. The citations of left, retired or deceased members of staff also pose problems of counting and allocation. Einstein last year had more than 800 citations. How are future citesums of recent work to be counted?

If citefaction and stupefaction are to be avoided, every academic should be ready to argue strongly against every suggestion that university citesums have any significance for evaluation.

G.G. HALL

Division of Molecular Engineering,

Graduate School of Engineering,

Kyoto University, Kyoto, Japan 